\title{
Infinite transitivity on the Calogero-Moser space $\mathcal{C}_{2}{ }^{*}$
}

\author{
J. Kesten, S. Mathers, and Z. Normatov
}

\author{
Communicated by V. M. Futorny
}

ABSTRACT. We prove a particular case of the conjecture of Berest-Eshmatov-Eshmatov by showing that the group of unimodular automorphisms of $\mathbb{C}[x, y]$ acts in an infinitely-transitive way on the Calogero-Moser space $\mathcal{C}_{2}$.

\section{Introduction}

Let $M_{n}$ be the $\mathbb{C}$-algebra of $n \times n$ matrices over $\mathbb{C}$. The group $\operatorname{GL}_{n}(\mathbb{C})$ acts on the direct product $M_{n} \times M_{n}$ in the natural way:

$$
g \cdot(X, Y)=\left(g X g^{-1}, g Y g^{-1}\right), \quad g \in \mathrm{GL}_{n}(\mathbb{C}) .
$$

For an integer $n \geqslant 0$, let $\hat{\mathcal{C}}_{n}$ be the subset of $M_{n} \times M_{n}$ defined as

$$
\left\{(X, Y) \in M_{n} \times M_{n}: \operatorname{rank}\left([X, Y]+I_{n}\right)=1\right\},
$$

where $I_{n}$ is the $n \times n$ identity matrix. The action of (1) on $M_{n} \times M_{n}$ restricts to an action on $\hat{\mathcal{C}}_{n}$, and we can then define the $n$-th CalogeroMoser space $\mathcal{C}_{n}$ to be the quotient $\hat{\mathcal{C}}_{n} / / \mathrm{GL}_{n}$. These spaces were studied in detail by Wilson [4], where it was shown, among other things, that $\mathcal{C}_{n}$ is a smooth, affine, irreducible, complex, symplectic variety of dimension $2 n$.

${ }^{*}$ The authors are indebted to Farkhod Eshmatov for proposing this problem and giving invaluable suggestions and would like to thank the International Research Experience for Undergraduates, organized through UC Fullerton and the Uzbekistan Academy of Sciences, for making the collaboration and project possible. Kesten and Mathers were supported by NSF Grant 1658672.

2020 MSC: 14R20, 14L30, 14J50.

Key words and phrases: Calogero-Moser space, infinite transitivity. 
The group of unimodular automorphisms of $\mathbb{C}[x, y]$ acts on $\mathcal{C}_{n}$, and it is proved in [1] that this action is doubly transitive. Additionally, a conjecture that this action is infinitely transitive is stated. Recently, in [3], this conjecture was proved.

The goal of this paper is to give another proof of infinite transitivity for the case $n=2$. The proofs here are more constructive and shed more light on the action on $\mathcal{C}_{2}$. We do this inductively by first choosing distinct points $x_{1}, \ldots, x_{n}, x_{n+1} \in \mathcal{C}_{2}$. Then, for any tuple of distinct elements $\left(y_{1}, \ldots, y_{n}, y_{n+1}\right)$, we use the inductive hypothesis to move $\left(y_{1}, \ldots, y_{n}, y_{n+1}\right)$ to $\left(x_{1}, \ldots, x_{n}, \tilde{y}_{n+1}\right)$. If we can then find elements of $G$ that stabilize $x_{1}, \ldots, x_{n}$ while acting transitively on the rest of the elements of $\mathcal{C}_{2}$, we can then move the element $\tilde{y}_{n+1}$ to the predetermined element $x_{n+1}$, while keeping $x_{1}, \ldots, x_{n}$ fixed. This will show that any tuple $\left(y_{1}, \ldots, y_{n+1}\right)$ is in the same orbit as $\left(x_{1}, \ldots, x_{n+1}\right)$, thus establishing $(n+1)$-transitivity. For this approach to work, we see that we will require information about the stabilizers of specific elements in $\mathcal{C}_{2}$, which we collect in future sections.

In general, an explicit representation for the coordinate ring, $\mathbb{C}\left[\mathcal{C}_{n}\right]$, of a Calogero-Moser space is not known. However, for $n=2$, it is not difficult to find. Let $A=X-\frac{1}{2} \operatorname{Tr}(X) I_{2}$ and $B=Y-\frac{1}{2} \operatorname{Tr}(Y) I_{2}$ be traceless matrices associated to $X$ and $Y$, respectively. In this case, using the generators $\left\{\operatorname{Tr}(X), \operatorname{Tr}(Y), \operatorname{Tr}\left(X^{2}\right), \operatorname{Tr}(X Y), \operatorname{Tr}\left(Y^{2}\right)\right\}$ of $\mathbb{C}\left[\left(M_{2} \times M_{2}\right) / / \mathrm{GL}_{2}\right]$ found in $[2]$, we define the following generators of $\mathbb{C}\left[\mathcal{C}_{2}\right]$ :

$$
a_{1}=\operatorname{Tr}(X), a_{2}=\operatorname{Tr}(Y), a_{3}=\operatorname{Tr}\left(A^{2}\right), a_{4}=\operatorname{Tr}(A B), a_{5}=\operatorname{Tr}\left(B^{2}\right) .
$$

Using the fact that a non-zero $2 \times 2$ matrix is of rank one if and only if its determinant is zero, we find that

$$
\mathbb{C}\left[\mathcal{C}_{2}\right]=\mathbb{C}\left[a_{1}, a_{2}, a_{3}, a_{4}, a_{5}\right] /\left(a_{4}^{2}-a_{3} a_{5}-1\right) .
$$

Note that there is a one to one correspondence between a point $(X, Y) \in \mathcal{C}_{2}$ and a point $\left(a_{1}, \ldots, a_{5}\right) \in \mathbb{C}^{5}$ such that $a_{4}^{2}-a_{3} a_{5}=1$, given by

$$
(X, Y) \mapsto\left(\operatorname{Tr}(X), \operatorname{Tr}(Y), \operatorname{Tr}\left(A^{2}\right), \operatorname{Tr}(A B), \operatorname{Tr}\left(B^{2}\right)\right) .
$$

\section{Preliminaries}

Denote by $G$ the group generated by the following two kinds of automorphisms of $M_{n} \times M_{n}$ :

(i) $\Phi_{p}:(X, Y) \mapsto(X, Y+p(X))$, where $p \in \mathbb{C}[t]$, 
(ii) $\Psi_{q}:(X, Y) \mapsto(X+q(Y), Y)$, where $q \in \mathbb{C}[t]$.

It is known [5], $\mathrm{G}$ is isomorphic to

$$
\operatorname{SAut}(C[x, y])=\left\{f=\left(f_{1}, f_{2}\right) \in \operatorname{Aut}(C[x, y]) \mid \operatorname{Jac}\left(f_{1}, f_{2}\right)=1\right\}
$$

where $\operatorname{Jac}\left(f_{1}, f_{2}\right)$ is the determinant of the Jacobian matrix of the map $\left(f_{1}, f_{2}\right)$. Note that Aut $(\mathbb{C}[x, y])$ is isomorphic to a semidirect product SAut $(\mathbb{C}[x, y]) \rtimes G_{m}$, where $G_{m}$ is a multiplicative group of the field $\mathbb{C}$ which acts on $\mathbb{C}[x, y]$ by scalar multiplication on variables $x$ and $y$. From the correspondence given in (2), we obtain an easy way of computing the action of the above group, $G$, using the following component-wise rules:

$$
\begin{aligned}
& \Phi_{p}\left(a_{1}\right):=a_{1} \\
& \Phi_{p}\left(a_{2}\right):=a_{2}+\operatorname{Tr}(p(X)) \\
& \Phi_{p}\left(a_{3}\right):=a_{3} \\
& \Phi_{p}\left(a_{4}\right):=a_{4}+\operatorname{Tr}(A p(X)) \\
& \Phi_{p}\left(a_{5}\right):=a_{5}+\operatorname{Tr}\left(p^{2}(X)\right)+2 \operatorname{Tr}(B \cdot p(X))-\frac{1}{3} \operatorname{Tr}^{2}(p(X)) .
\end{aligned}
$$

The action of $\Psi_{q}$ on $\mathcal{C}_{2}$ is similar, and is symmetric to (3).

For a matrix $M=\left(\begin{array}{ll}\alpha & \beta \\ \lambda & \mu\end{array}\right) \in \mathrm{SL}_{2}$ (so that $\alpha \mu-\beta \lambda=1$ ) consider $\Theta_{M}: \mathcal{C}_{2} \rightarrow \mathcal{C}_{2}$ defined by

$$
(X, Y) \mapsto(\alpha X+\beta Y, \lambda X+\mu Y) .
$$

One can easily find that the action $\Theta_{M}$ is a composition of the automorphisms of type (i) and (ii) using some linear polynomials $p$ and $q$. Under this action, a point will change as follows:

$$
\begin{aligned}
& \Theta_{M}\left(a_{1}\right)=\alpha a_{1}+\beta a_{2} \\
& \Theta_{M}\left(a_{2}\right)=\lambda a_{1}+\mu a_{2} \\
& \Theta_{M}\left(a_{3}\right)=\alpha^{2} a_{3}+2 \alpha \beta a_{4}+\beta^{2} a_{5} \\
& \Theta_{M}\left(a_{4}\right)=\alpha \lambda a_{3}+(\alpha \mu+\beta \lambda) a_{4}+\beta \mu a_{5} \\
& \Theta_{M}\left(a_{5}\right)=\lambda^{2} a_{3}+2 \lambda \mu a_{4}+\mu^{2} a_{5}
\end{aligned}
$$

We now remind the following definitions concerning group actions on sets. To do this, let $\mathcal{G}$ be a group acting on a set $S$.

Definition 1. We say the group $\mathcal{G}$ acts transitively, or that the action is transitive, if for every pair of elements $s, r \in S$, there is a $g \in \mathcal{G}$ such that $g \cdot s=r$. 
Definition 2. The group $\mathcal{G}$ acts $n$-transitively, or the action is $n$-transitive, if it can map any $n$-tuple of distinct points of the set to any other $n$-tuple of distinct points. In other words, if $\left(s_{1}, \ldots, s_{n}\right)$ and $\left(r_{1}, \ldots, r_{n}\right)$ are $n$ tuples of distinct elements in $S$, then there is some $g \in \mathcal{G}$ such that $g \cdot\left(s_{1}, \ldots, s_{n}\right)=\left(g \cdot s_{1}, \ldots, g \cdot s_{n}\right)=\left(r_{1}, \ldots, r_{n}\right)$.

Definition 3. Lastly, we say that the action of $\mathcal{G}$ on $S$ is infinitely transitive if it is $n$-transitive for every positive integer $n$.

We now claim that the action of $G$ on $\mathcal{C}_{2}$ defined above is infinitelytransitive.

\section{Base cases: $n=1,2,3$}

As stated previously, we plan to prove this main result by induction, and so we begin by proving the base cases for $n=1,2,3$. We start with $n=1$ :

Proposition 1. The action of $G$ on $\mathcal{C}_{2}$ is a transitive group action.

Proof. Let $A=\left(a_{1}, a_{2}, a_{3}, a_{4}, a_{5}\right) \in \mathcal{C}_{2}$ be an arbitrary point. Note that this proof does not require us to stabilize any elements, and so we may use $p(t)=-\frac{a_{2}}{2}$ and $q(t)=-\frac{a_{1}}{2}$, to get that

$$
\left(\Psi_{q} \circ \Phi_{p}\right)(A)=\left(0,0, a_{3}, a_{4}, a_{5}\right) .
$$

From here we use the action of $\Theta_{M}$, defined in (4), with either the matrix $M_{+}:=\left[\begin{array}{cc}-\frac{a_{5}}{2\left(a_{4}+1\right)} & \frac{1}{2} \\ a_{4}+1 & a_{3}\end{array}\right]$ or the matrix $M_{-}:=\left[\begin{array}{cc}-\frac{a_{5}}{2\left(a_{4}-1\right)} & \frac{1}{2} \\ a_{4}-1 & a_{3}\end{array}\right]$ to reach the point

$$
\Theta_{M}\left(0,0, a_{3}, a_{4}, a_{5}\right)=(0,0,0,1,0) .
$$

More specifically, if $a_{3}=0$ or $a_{5}=0$, then, since $a_{4}^{2}-a_{3} a_{5}=1$, we must have that $a_{4}= \pm 1$. If $a_{4}=1$, then we use the matrix $M_{+}$. If $a_{4}=-1$, we use the matrix $M_{-}$. If $a_{3} a_{5} \neq 0$, then either matrix $M_{+}$or $M_{-}$will suffice. Thus we have that all elements $A \in \mathcal{C}_{2}$ are in the orbit of the point $(0,0,0,1,0) \in \mathcal{C}_{2}$.

Next, we prove 2- and 3-transitivity, since they differ from the general $n$ case by requiring us only to focus on stabilizing nilpotent points. We will need the following two lemmas:

Lemma 1. Let $A \in \mathcal{C}_{2} \backslash\{(0,0,0, \pm 1,0)\}$. Then there is a $g \in$ $\operatorname{Stab}\{(0,0,0, \pm 1,0)\}$ such that $A^{\prime}=g A$ satisfies $a_{1}^{\prime} a_{3}^{\prime} \neq 0$, where $A^{\prime}=$ $\left(a_{1}^{\prime}, a_{2}^{\prime}, a_{3}^{\prime}, a_{4}^{\prime}, a_{5}^{\prime}\right)$. 
Proof. We may assume at least one of $a_{1}, a_{2}, a_{3}, a_{5}$ is nonzero. We will proceed by case work.

Case 1: $a_{1} \neq 0$. If $a_{3} \neq 0$ we are already done, so suppose $a_{3}=0$. Without loss of generality, we may assume that $a_{2} \neq 0$, since if $a_{2}=0$, we may apply $\Phi_{t^{2}}$ to arrive at the point $\left(b_{1}, b_{2}, b_{3}, b_{4}, b_{5}\right)$ with $b_{2}=\frac{a_{1}^{2}}{2} \neq 0$. Additionally, since $a_{3}=0$, we must have $a_{4}$ is non-zero, so that there exists an $\alpha \in \mathbb{C}$ such that $\alpha a_{2}\left(\alpha a_{2} a_{5}+2 a_{4}\right) \neq 0$ and such that $\alpha\left(a_{5}+\frac{a_{2}^{2}}{2}\right)+a_{1} \neq 0$, since this is a non-zero polynomial in $\alpha$. We now apply $\Psi_{\alpha t^{2}}$ to arrive at $A^{\prime}$ where $a_{1}^{\prime} a_{3}^{\prime} \neq 0$.

Case 2: $a_{1}=0$. From here, we will show that we can move into case one.

Case 2.1: $a_{5}+\frac{a_{2}^{2}}{2} \neq 0$. We can calculate explicitly that applying $\Psi_{t^{2}}$ gives $a_{1}^{\prime}=a_{5}+\frac{a_{2}^{2}}{2} \neq 0$, so that we are back in Case 1 .

Case 2.2: $a_{5}+\frac{a_{2}^{2}}{2}=0$.

Case 2.2.1: $a_{3} \neq 0$. We can map $a_{2}$ and $a_{5}$ to $a_{2}^{\prime}$ and $a_{5}^{\prime}$ such that $a_{5}^{\prime}+\frac{\left(a_{2}^{\prime}\right)^{2}}{2} \neq 0$ by $\Phi_{\beta t^{2}}$, since $a_{2}^{\prime}=a_{2}+\beta a_{3}$ and $a_{5}^{\prime}=a_{5}$. This moves us back to Case 2.1.

Case 2.2.2: $a_{3}=0$. Since $a_{1}=0$ and $a_{5}+\frac{a_{2}^{2}}{2}=0$ with $a_{2} \neq 0$ after we are at the point $\left(0, a_{2}, 0, \pm 1, \frac{-a_{2}^{2}}{2}\right)$. By applying $\Psi_{t^{3}}$, we can send $a_{1}$ to $\frac{-a_{2}^{3}}{2}$, thus showing that we can send $a_{1}$ to a nonzero value, returning us to Case 1 and completing the proof. It is easy to check that all of the elements of $G$ used above are indeed in $\operatorname{Stab}\{(0,0,0, \pm 1,0)\}$.

Lemma 2. Let $A \in \mathcal{C}_{2}$ with $a_{1} a_{3} \neq 0$. Then there is a $g \in$ $\operatorname{Stab}\{(0,0,0, \pm 1,0)\}$ such that $A^{\prime}=g A$ satisfies $a_{1}^{\prime} a_{3}^{\prime} \neq 0$ and $a_{3}^{\prime} \neq \frac{a_{1}^{2}}{2}$.

Proof. Let $A=\left(a_{1}, a_{2}, a_{3}, a_{4}, a_{5}\right)$ with $a_{1} a_{3} \neq 0$ be given. We also assume $a_{3}=\frac{a_{1}^{2}}{2}$, since otherwise we are done.

Case 1: $a_{2} a_{5} \neq 0$. Then we can apply $\Psi_{\alpha t^{2}}$ to get

$$
\begin{aligned}
\Psi_{\alpha t^{2}}\left(a_{1}, a_{2}, \frac{a_{1}^{2}}{2}, a_{4}, a_{5}\right)= & \left(a_{1}+\left(\frac{a_{2}}{2}+a_{5}\right) \alpha, a_{2}, \frac{a_{1}^{2}}{2}+2 a_{2} a_{4} \alpha+a_{2}^{2} a_{5} \alpha^{2},\right. \\
& \left.a_{4}+a_{2} a_{5} \alpha, a_{5}\right) .
\end{aligned}
$$

From this we can see that $a_{1}^{\prime}, a_{3}^{\prime}$ are non-zero polynomials of $\alpha$, so that there are at most finitely many values of $\alpha$ such that $a_{1}^{\prime} a_{3}^{\prime}=0$. Additionally, plugging into $a_{1}^{\prime 2}-2 a_{3}^{\prime}$, we obtain the polynomial

$$
\left(-4 a_{2} a_{4}+2 a_{1}\left(a_{2}^{2} / 2+a_{5}\right)\right) \alpha+\left(-2 a_{2}^{2} a_{5}+\left(a_{2}^{2} / 2+a_{5}\right)^{2}\right) \alpha^{2} .
$$


We claim this is a non-zero polynomial in $\alpha$. To see this, assume that it is the zero polynomial, so that $-4 a_{2} a_{4}+2 a_{1}\left(a_{2}^{2} / 2+a_{5}\right)=0$ and $-2 a_{2}^{2} a_{5}+\left(a_{2}^{2} / 2+a_{5}\right)^{2}=0$. This implies that $a_{5}=\frac{-a_{1} a_{2}^{2}+4 a_{2} a_{4}}{2 a_{1}}$ and $a_{5}=\frac{a_{2}^{2}}{2}$. Setting these equal means that we must have $a_{4}=\frac{a_{1} a_{2}}{2}$. (Note that this is where we have used the assumption that $a_{2} a_{5} \neq 0$, so that we can actually solve for $a_{4}$ in this way.) It leads to a contradiction. Thus we have that $a_{1}^{\prime 2}-2 a_{3}^{\prime}$ is a non-zero polynomial in $\alpha$, and hence, since there are uncountably many $\alpha \in \mathbb{C}$, we can choose an $\alpha$ such that $a_{1}^{\prime} a_{3}^{\prime} \neq 0$ and $a_{1}^{\prime 2}-2 a_{3}^{\prime} \neq 0$, as desired.

Case 2: $a_{2} a_{5}=0$. We again assume that $a_{3}=\frac{a_{1}^{2}}{2}$. Applying $\Phi_{\alpha t^{2}}$, we get

$\Phi_{\alpha t^{2}}\left(a_{1}, a_{2}, \frac{a_{1}^{2}}{2}, a_{4}, a_{5}\right)=\left(a_{1}, a_{2}+a_{1}^{2} \alpha, \frac{a_{1}^{2}}{2}, a_{4}+\frac{a_{1}^{3} \alpha}{2}, a_{5}+2 a_{1} a_{4} \alpha+\frac{a_{1}^{4} \alpha^{2}}{2}\right)$

From this, since $a_{1} \neq 0$, we can conclude that $a_{2}^{\prime}$ and $a_{5}^{\prime}$ are non-zero polynomials in $\alpha$, and hence we can choose some $\alpha \in \mathbb{C}$ such that $a_{2}^{\prime} a_{5}^{\prime} \neq 0$, landing us back in the Case 1. (Again, it is easy to check that all elements of $G$ used in the above proof are elements of $\operatorname{Stab}\{(0,0,0, \pm 1,0)\}$.)

We are now able to prove 2- and 3- transitivity:

Proposition 2. The action of $G$ on $\mathcal{C}_{2}$ is a 2-transitive group action.

Proof. Let $(A, B) \in \mathcal{C}_{2} \times \mathcal{C}_{2}$ be a pair of distinct points. Then, since $G$ acts on $\mathcal{C}_{2}$ transitively by Proposition 1 , there is a $g \in G$ such that $g(A, B)=\left((0,0,0,1,0), B^{\prime}\right)$ for some $B^{\prime} \in \mathcal{C}_{2}$. Thus, if there is an $h \in$ $\operatorname{Stab}\{(0,0,0,1,0)\}$ such that $h B^{\prime}=(0,0,0,-1,0)$, we are done. In particular, this reduces the problem to showing that for any $A \in \mathcal{C}_{2} \backslash\{(0,0,0,1,0)\}$, there is a $g \in \operatorname{Stab}\{(0,0,0,1,0)\}$ such that $g A=(0,0,0,-1,0)$.

Thus, let $A \in \mathcal{C}_{2} \backslash\{(0,0,0,1,0)\}$ be an arbitrary point. If $A=(0,0,0,-1,0)$, then we are already at the point we desire. Otherwise, we have that $A \in \mathcal{C}_{2} \backslash\{(0,0,0, \pm 1,0)\}$. Using Lemmas 1 and 2, we may also assume that $a_{1} a_{3} \neq 0$ and $a_{1}^{2}-2 a_{3} \neq 0$. Applying $\Phi_{\alpha t^{3}} \in$ $\operatorname{Stab}\{(0,0,0,1,0)\}$ to $A$, we reach the point

$$
\begin{aligned}
A^{\prime}=\left(a_{1}, a_{2}+\left(a_{1} a_{3}+\frac{1}{2} a_{1}\left(\frac{a_{1}^{2}}{2}+a_{3}\right)\right) \alpha, a_{3},\right. \\
a_{4}+\left(\frac{a_{1}^{2} a_{3}}{2}+\frac{1}{2} a_{3}\left(\frac{a_{1}^{2}}{2}+a_{3}\right)\right) \alpha, \\
\left.a_{5}+\left(\frac{3 a_{1}^{2}}{2}+a_{3}\right) a_{4} \alpha+\frac{1}{4} a_{3}\left(\frac{3 a_{1}^{2}}{2}+a_{3}\right)^{2} \alpha^{2}\right) .
\end{aligned}
$$


Then we can calculate that

$$
\begin{aligned}
& -4 a_{1}^{\prime 3} a_{2}^{\prime} a_{4}^{\prime}-8 a_{1}^{\prime} a_{2}^{\prime} a_{3}^{\prime} a_{4}^{\prime}+a_{1}^{\prime 4} a_{5}^{\prime}+4 a_{3}^{\prime 2} a_{5}^{\prime}+4 a_{1}^{\prime 2} a_{3}^{\prime}\left(a_{2}^{\prime 2}+a_{5}^{\prime}\right) \\
& =4 a_{1}^{2} a_{2}^{2} a_{3}-4 a_{1}^{3} a_{2} a_{4}-8 a_{1} a_{2} a_{3} a_{4}+a_{1}^{4} a_{5}+4 a_{1}^{2} a_{3} a_{5}+4 a_{3}^{2} a_{5} \\
& +\left(-a_{1}^{5} a_{2} a_{3}+4 a_{1}^{3} a_{2} a_{3}^{2}-4 a_{1} a_{2} a_{3}^{3}+\frac{a_{1}^{6} a_{4}}{2}-a_{1}^{4} a_{3} a_{4}\right. \\
& \left.\quad-2 a_{1}^{2} a_{3}^{2} a_{4}+4 a_{3}^{3} a_{4}\right) \alpha+\left(\frac{a_{1}^{8} a_{3}}{16}-\frac{a_{1}^{6} a_{3}^{2}}{2}+\frac{3 a_{1}^{4} a_{3}^{3}}{2}-2 a_{1}^{2} a_{3}^{4}+a_{3}^{5}\right) \alpha^{2} .
\end{aligned}
$$

One can check that if the coefficient of the $\alpha^{2}$ term is zero, then either $a_{3}=0$ or $a_{3}=a_{1}^{2} / 2$. Since we know neither of these is true, it follows that the last term is non-zero, and hence this is a non-zero polynomial in $\alpha$. Thus we can choose a $\alpha \in \mathbb{C}$ such that this polynomial does not vanish. Since this is the case, we can consider the polynomials

$$
p(t)=\frac{-a_{4}^{\prime}+1}{a_{1}^{\prime} a_{3}^{\prime}} t^{2}=\frac{-a_{4}^{\prime}+1}{a_{1} a_{3}} t^{2}
$$

and

$$
q(t)=\frac{a_{1}^{\prime} a_{3}^{\prime}\left(2 a_{1}^{\prime} a_{2}^{\prime} a_{3}^{\prime}-a_{1}^{\prime 2}\left(a_{4}^{\prime}-1\right)-2 a_{3}^{\prime}\left(a_{4}^{\prime}-1\right)\right)}{-4 a_{1}^{\prime 3} a_{2}^{\prime} a_{4}^{\prime}-8 a_{1}^{\prime} a_{2}^{\prime} a_{3}^{\prime} a_{4}^{\prime}+a_{1}^{\prime 4} a_{5}^{\prime}+4 a_{3}^{\prime 2} a_{5}^{\prime}+4 a_{1}^{\prime 2} a_{3}^{\prime}\left(a_{2}^{\prime 2}+a_{5}^{\prime}\right)} t^{2} .
$$

These satisfy $\Phi_{p(t)}, \Psi_{q(t)} \in \operatorname{Stab}\{(0,0,0,1,0)\}$, and we can calculate that

$$
\left(\Psi_{q} \circ \Phi_{p}\right)\left(a_{1}^{\prime}, a_{2}^{\prime}, a_{3}^{\prime}, a_{4}^{\prime}, a_{5}^{\prime}\right)=\left(b_{1}, b_{2}, 0,-1,0\right),
$$

for some $b_{1}, b_{2} \in \mathbb{C}$.

Case 1: $b_{1} b_{2} \neq 0$. Using $\lambda=\frac{b_{1}}{b_{2}}$ and $\mu=\frac{b_{2}^{2}}{4 b_{1}}$, we can apply the following composition to get

$$
\left(\Psi_{\lambda t} \circ \Phi_{\mu t^{2}+\lambda \mu} \circ \Psi_{-\lambda t}\right)\left(b_{1}, b_{2}, 0,-1,0\right)=(0,0,0,-1,0),
$$

as desired. One can easily check that this composition is in $\operatorname{Stab}\{(0,0,0,1,0)\}$.

Case 2: $b_{1} b_{2}=0$. If $b_{1}=b_{2}=0$, then we already have that $\left(b_{1}, b_{2}, 0,-1,0\right)=(0,0,0,-1,0)$. If not, we have that either $b_{1} \neq 0$ or $b_{2} \neq 0$. In these cases we use the element $\Phi_{t^{2}-\frac{4}{3 a_{1}^{\prime}} t^{3}}$ or $\Psi_{t^{2}-\frac{4}{3 a_{2}^{\prime}}}$, respectively, in order to map

$$
\left(b_{1}, 0,0,-1,0\right) \mapsto\left(b_{1}, b_{1}^{2} / 6,0,-1,0\right),
$$


or

$$
\left(0, b_{2}, 0,-1,0\right) \mapsto\left(b_{2}^{2} / 6, b_{2}, 0,-1,0\right),
$$

landing us back in Case 1.

Thus we have shown that all points in $\mathcal{C}_{2} \backslash(0,0,0,1,0)$ are in the same orbit as $(0,0,0,-1,0)$ under the action of $\operatorname{Stab}\{(0,0,0,1,0)\}$, so that $G$ acts 2-transitively on $\mathcal{C}_{2}$.

Proposition 3. The action of $G$ on $\mathcal{C}_{2}$ is a 3 -transitive group action.

Proof. Since the group $G$ acts 2-transitively by Proposition 2, we can reduce the problem to showing that for any $A \in C_{2} \backslash\{(0,0,0, \pm 1,0)\}$, there is some $g \in S=\operatorname{Stab}\{(0,0,0, \pm 1,0)\}$ such that $g(A)=(0,0,0,-1,2)$. A straightforward computation shows that all of the elements of $\operatorname{Stab}\{(0,0,0,1,0)\}$ used in the proof of Proposition (2) also stabilize the point $(0,0,0,-1,0)$, so that, using Lemmas 1 and 2 , and then proceeding analogously to the proof of Proposition 2, we are able to find some $g \in S$ such that $g(A)=\left(b_{1}, b_{2}, 0,-1,0\right)$.

We may assume that $b_{1}$ and $b_{2}$ are not simultaneously zero.

Case 1: $b_{1} \neq 0$. For the polynomials $p_{1}(t)=\frac{4\left(b_{1}-4 b_{2}\right)}{b_{1}^{3}} t^{3}-\frac{8\left(b_{1}-3 b_{2}\right)}{b_{1}^{4}} t^{4}$ and $q_{1}(t)=\frac{-b_{1}}{2} t^{2}$, we get that $\left(\Psi_{q_{1}} \circ \Phi_{p_{1}}\right) \in \operatorname{Stab}\{(0,0,0, \pm 1,0)\}$ and

$$
\left(\Psi_{q_{1}} \circ \Phi_{p_{1}}\right)\left(b_{1}, b_{2}, 0,-1,0\right)=(0,0,0,-1,2),
$$

as desired.

Case 2: $b_{2} \neq 0$. Similarly, using the polynomials $p_{2}(t)=\frac{-b_{2}}{2} t^{2}$ and $q_{2}(t)=\frac{4\left(b_{2}-4 b_{1}\right)}{b_{2}^{3}} t^{3}-\frac{8\left(b_{2}-3 b_{1}\right)}{b_{2}^{4}} t^{4}$, we obtain that $\left(\Phi_{p_{2}} \circ \Psi_{q_{2}}\right) \in$ $\operatorname{Stab}\{(0,0,0, \pm 1,0)\}$ and

$$
\left(\Phi_{p_{2}} \circ \Psi_{q_{2}}\right)\left(b_{1}, b_{2}, 0,-1,0\right)=(0,0,2,-1,0) .
$$

Then, we can apply $\left(\Psi_{t^{3}} \circ \Phi_{\frac{1}{3} t^{2}}\right) \in \operatorname{Stab}(0,0,0, \pm 1,0)$ to get to the point

$$
\left(\Psi_{t^{3}} \circ \Phi_{\frac{1}{3} t^{2}}\right)(0,0,2,-1,0)=(2 / 3,2,0,-1,0),
$$

landing us back in the case where $b_{1} \neq 0$.

\section{Stabilizer elements}

While proving the base cases, we could easily check that the elements of $G$ being used were in the desired stabilizers; unfortunately it is not as easy to do this as the sets of points we wish to stabilize get larger. This section is concerned with determining which elements of $G$ are in the stabilizers of larger subsets of $\mathcal{C}_{2}$. 
Proposition 4. Let $A=\left(0,0,0, \pm 1, a_{5}\right)$ be a point in $\mathcal{C}_{2}$. Then $\Phi_{p}$ stabilizes $A$ if and only if $t^{2} \mid p(t)$.

Proof. Let $(X, Y)$ be the pair of matrices in $\mathcal{C}_{2}$ that corresponds to the point $A$, and recall that the action of $\Phi_{p}$ on $A$ corresponds to the action on $(X, Y)$ defined by $\Phi_{p}(X, Y)=(X, Y+p(X))$ in $\mathcal{C}_{2}$.

Assume $t^{2} \mid p(t)$ and let us show that $\Phi_{p}(A)=A$. Since $\Phi_{p} \circ \Phi_{q}=\Phi_{p+q}$, it is enough to show that for any monomial $\alpha t^{n}$ with $n \geqslant 2$ and $\alpha \in \mathbb{C}$, we have that $\Phi_{\alpha t^{n}}$ stabilizes $A$. Since $a_{1}=a_{3}=0$, we can conclude that $X$ is nilpotent, meaning that $X^{n}=0$ for any $n \geqslant 2$. It follows that $p(X)=\alpha X^{n} \equiv 0$, so that $\Phi_{p}(X, Y)=(X, Y+p(X))=(X, Y)$, and hence $\Phi_{p}(A)=A$.

Conversely, suppose that $t^{2} \nmid p(t)$. This implies that $p$ has non-zero linear or constant terms. Also, since $a_{1}=a_{3}=0, X$ is still nilpotent, so that we can assume $p(t)=\alpha t+\beta$ where one of the parameters $\alpha, \beta$ is nonzero. Then, using (3), we have that

$$
\Phi_{p}(A)=\left(0,2 \beta, 0, \pm 1, a_{5} \pm 2 \alpha\right),
$$

so that $\Phi_{p}$ does not fix $A$, thus proving the contrapositive.

Now, consider the point $A=\left(0,0,0,-1,2^{k}\right)$ for some $k \in \mathbb{Z}_{+}$. We want to determine which $q \in \mathbb{C}[t]$ will satisfy $\Psi_{q}(A)=A$. Again, since $a_{1}=a_{3}=0, X$ is nilpotent, and hence we may assume that $X=\left(\begin{array}{ll}0 & 1 \\ 0 & 0\end{array}\right)$. Also, $\operatorname{Tr}(Y)=0$, so that we can write $Y=\left(\begin{array}{cc}a & b \\ c & -a\end{array}\right)$.

Next, we note that $\operatorname{Tr}(X Y)=-1$, which implies that $c=-1$. Now we note that under the group action of $\mathrm{GL}_{2}$ by the matrix $M=\left(\begin{array}{ll}1 & a \\ 0 & 1\end{array}\right)$, we may assume that $a=0$, since $M X M^{-1}=X$ and $M Y M^{-1}=\left(\begin{array}{cc}0 & b^{\prime} \\ -1 & 0\end{array}\right)$. Thus, we have that $Y=\left(\begin{array}{cc}0 & b \\ -1 & 0\end{array}\right)$ in $\mathcal{C}_{2}$.

Lastly, consider the fact that $\operatorname{Tr}\left(Y^{2}\right)=2^{k}$, we have $b=-2^{k-1}$, giving us $Y=\left(\begin{array}{cc}0 & -2^{k-1} \\ -1 & 0\end{array}\right)$.

Now that we have nice formulas for $X$ and $Y$, we can explicitly see how our group action, defined by $(X, Y) \mapsto(X+q(Y), Y)$, acts on this specific pair of matrices. We wish to determine which $q \in \mathbb{C}[t]$ will satisfy 
$X+q(Y)=X$, thus stabilizing the point $\left(0,0,0,-1,2^{k}\right)$. We start with $q(t)=\sum_{i=1}^{n} \alpha_{2 i} t^{2 i}$. Then we have that

$$
X \mapsto X+\sum_{i=1}^{n} \alpha_{2 i}\left(2^{k-1}\right)^{i} I_{2} .
$$

Thus, to stabilize the point, we must have that $\sum_{i=1}^{n}\left(2^{k-1}\right)^{i} \alpha_{2 i}=0$.

A similar argument shows that if $q(t)=\sum_{i=1}^{n} \alpha_{2 i+1} t^{2 i+1}$, then we must have that $\sum_{i=1}^{n}\left(2^{k-1}\right)^{i} \alpha_{2 i+1}=0$.

Therefore, concerning the set of points

$$
\left\{(0,0,0, \pm 1,0),(0,0,0,-1,2),(0,0,0,-1,4), \cdots,\left(0,0,0,-1,2^{k}\right)\right\}
$$

for some $k \in \mathbb{Z}_{+}$, if we have that $\sum_{i=1}^{n}\left(2^{j-1}\right)^{i} \alpha_{2 i}=0$ and $\sum_{i=1}^{n}\left(2^{j-1}\right)^{i} \alpha_{2 i+1}=0$ for all $1 \leqslant j \leqslant k$, then all the above points will be stabilized under the action of $\Psi_{q}$. This brings us to the following Lemma, which deals with finding solutions to this necessary system of equations obtained from the previous discussion:

Lemma 3. The solution set to the system of equations given by

$$
\left\{\begin{array}{l}
a_{1}+a_{2}+\ldots+a_{n}=0 \\
2 a_{1}+2^{2} a_{2}+\ldots+2^{n} a_{n}=0 \\
4 a_{1}+4^{2} a_{2}+\ldots+4^{n} a_{n}=0 \\
\vdots \\
2^{n-2} a_{1}+\left(2^{n-2}\right)^{2} a_{2}+\ldots+\left(2^{n-2}\right)^{n} a_{n}=0
\end{array}\right.
$$

can be expressed in terms of $a_{n}$ as

$$
\left\{\begin{array}{l}
a_{1}=S(n-1, n-2) a_{n} \\
a_{2}=S(n-2, n-2) a_{n} \\
a_{3}=S(n-3, n-2) a_{n} \\
\vdots \\
a_{n-1}=S(1, n-2) a_{n} \\
a_{n}=a_{n}
\end{array}\right.
$$

where $S(i, j)$ is the $i^{\text {th }}$ symmetric sum on the set $\left\{-1,-2,-4, \ldots,-2^{j}\right\}$.

Proof. The base of induction for $n=2$ is straightforward. 
For our inductive hypothesis, assume the claim is true for $n=k$, and let us consider the case $n=k+1$. We begin by noting that for $1 \leqslant i \leqslant k$, $S(i, k-1)=S(i-1, k-2)-2^{k-1} S(i, k-2)$.

If $\left(a_{1}, a_{2}, \ldots, a_{k}\right)$ is the solution set of the $(k-1)$-dimensional system, then by the inductive hypothesis we have that

$$
a_{i}=S(k-i, k-2) a_{k} \quad 1 \leqslant i \leqslant k-1 .
$$

Define $b_{i}$ for $1 \leqslant i \leqslant k+1$ such that $b_{i}=a_{i-1}-2^{k-1} a_{i}$, where $a_{0}$ and $a_{k+1}$ are defined to be 0 . Substituting in (5), we get that

$$
b_{i}=S(k-i+1, k-2) a_{k}-2^{k-1} S(k-i, k-2) a_{k}=S(i, k-1) a_{k}
$$

for $1 \leqslant i \leqslant k$. Furthermore, we get that $b_{k+1}=a_{k}$. Thus, to prove the claim, it suffices to show that

$$
\left(b_{1}, b_{2}, \ldots, b_{k+1}\right)=\left(0-2^{k-1} a_{1}, a_{1}-2^{k-1} a_{2}, \ldots, a_{i}-2^{k-1} a_{i+1}, \ldots, a_{k}\right)
$$

is a solution to the system of $k$ equations,

$$
\left\{\begin{array}{l}
x_{1}+x_{2}+\ldots+x_{k+1}=0 \\
2 x_{1}+2^{2} x_{2}+\ldots+2^{n} x_{k+1}=0 \\
4 x_{1}+4^{2} x_{2}+\ldots+4^{n} x_{k+1}=0 \\
\vdots \\
2^{k-1} x_{1}+\left(2^{k-1}\right)^{2} x_{2}+\ldots+\left(2^{k-1}\right)^{n} x_{k+1}=0
\end{array}\right.
$$

For the equation

$$
2^{i} x_{1}+\left(2^{i}\right)^{2} x_{2}+\ldots+\left(2^{i}\right)^{n} x_{k+1}=0,
$$

we plug in $b_{j}$ for $x_{j}$ to get

$$
\begin{aligned}
& 2^{i} b_{1}+\left(2^{i}\right)^{2} b_{2}+\ldots+\left(2^{i}\right)^{n} b_{k+1} \\
& \quad=2^{k-1}\left(-2^{i} a_{1}-2^{2 i} a_{2}-\ldots-2^{k i} a_{k}\right)+2^{i}\left(2^{i} a_{1}+2^{2 i} a_{2}+\ldots+2^{k i} a_{k}\right) .
\end{aligned}
$$

Now, for $i<k-1$, by the inductive hypothesis,

$$
2^{i} a_{1}+2^{2 i} a_{2}+\ldots+2^{j i} a_{j}+\ldots+2^{k i} a_{k}=0
$$

so both terms become 0 . For $i=k-1$, we have by direct substitution that

$$
\begin{aligned}
& 2^{k-1}\left(-2^{k-1} a_{1}-2^{2(k-1)} a_{2}-\ldots-2^{j(k-1)} a_{j}-\ldots-2^{k(k-1)} a_{k}\right) \\
& \quad+2^{k-1}\left(2^{k-1} a_{1}+2^{2(k-1)} a_{2}+\ldots+2^{j(k-1)} a_{j}+\ldots+2^{k(k-1)} a_{k}\right)=0 .
\end{aligned}
$$

Thus, we can conclude that $\left(b_{1}, b_{2}, \ldots, b_{k+1}\right)$ satisfy the system of equations for $n=k+1$, and since $b_{i}=S(i, k-1) a_{k}=S(i, k-1) b_{k+1}$, we are done. 
From this and the preceding discussion, we immediately conclude that if we wish to stabilize all the points

$$
\left\{(0,0,0, \pm 1,0),(0,0,0,-1,2),(0,0,0,-1,4), \cdots,\left(0,0,0,-1,2^{k}\right)\right\}
$$

using a polynomial with only even powers, we can use

$q_{k}^{E}(t):=\alpha t^{2(k+1)}+\alpha S(1, k-1) t^{2 k}+\cdots \alpha S(k-1, k-1) t^{4}+\alpha S(k, k-1) t^{2}$.

Similarly, if we wish to use a polynomial with only odd powers, we can use $q_{k}^{O}(t):=\alpha t^{2 k+3}+\alpha S(1, k-1) t^{2 k+1}+\cdots \alpha S(k-1, k-1) t^{5}+\alpha S(k, k-1) t^{3}$.

Notation. Let $q_{k}^{E}(t)$ and $q_{k}^{O}(t)$ be defined as above. We then define the following notation: $\Psi_{k}^{E}:=\Psi_{q_{k}^{E}}$ and $\Psi_{k}^{O}:=\Psi_{q_{k}^{O}}$.

From the above arguments, we can conclude that $\Psi_{k}^{E}$ and $\Psi_{k}^{O}$ will stabilize the set

$$
\left\{(0,0,0, \pm 1,0),(0,0,0,-1,2),(0,0,0,-1,4), \cdots,\left(0,0,0,-1,2^{k}\right)\right\} .
$$

Now, consider elements of the form $\Psi_{q} \circ \Phi_{p} \circ \Psi_{-q}$ and $\Phi_{p} \circ \Psi_{q} \circ \Phi_{-p}$, which we will call conjugation by $\Psi_{q}$ and $\Phi_{p}$, respectively. We will use the following four lemmas without proof, since the proofs are not difficult.

Lemma 4. The action $\Psi_{t} \circ \Phi_{p(t)} \circ \Psi_{-t}$ stabilizes the point $\left(0,0,0,1, a_{5}\right)$ if and only if $t^{2}-\frac{a_{5}-2}{2}$ divides $p(t)$ and it stabilizes $\left(0,0,0,-1, a_{5}\right)$ if and only if $t^{2}-\frac{a_{5}+2}{2}$ divides $p(t)$.

Lemma 5. The action $\Phi_{t} \circ \Psi_{q(t)} \circ \Phi_{-t}$ stabilizes the point $\left(0,0,0,1, a_{5}\right)$ if and only if $t^{2}-\frac{a_{5}-2}{2}$ divides $q(t)$ and it stabilizes $\left(0,0,0,-1, a_{5}\right)$ if and only if $t^{2}-\frac{a_{5}+2}{2}$ divides $q(t)$.

Lemma 6. The action $\Psi_{-t} \circ \Phi_{p(t)} \circ \Psi_{t}$ stabilizes the point $(0,0,0,1, a)$ if and only if $t^{2}-\frac{a+2}{2}$ divides $p(t)$ and it stabilizes $(0,0,0,-1, b)$ if and only if $t^{2}-\frac{b-2}{2}$ divides $p(t)$.

Lemma 7. The action $\Phi_{-t} \circ \Psi_{q(t)} \circ \Phi_{t}$ stabilizes the point $(0,0,0,1, a)$ if and only if $t^{2}-\frac{a+2}{2}$ divides $q(t)$ and it stabilizes $(0,0,0,-1, b)$ if and only if $t^{2}-\frac{b-2}{2}$ divides $q(t)$.

The following conjugations

$$
\begin{array}{ll}
\Phi_{t} \circ \Psi_{q_{k}^{C}} \circ \Phi_{-t} ; & \Psi_{t} \circ \Phi_{p_{k}^{C}} \circ \Psi_{-t} ; \\
\Phi_{-t} \circ \Psi_{\tilde{q}_{k}^{C}} \circ \Phi_{t} ; & \Psi_{-t} \circ \Phi_{\tilde{p}_{k}^{C}} \circ \Psi_{t} .
\end{array}
$$


will all stabilize the set

$$
\left\{(0,0,0, \pm 1,0),(0,0,0,-1,2), \cdots,\left(0,0,0,-1,2^{k}\right)\right\} \backslash\{(0,0,0,-1,8)\}
$$

where

$$
\begin{aligned}
& q_{k}^{C}(t)=\alpha\left(t^{2}+1\right)\left(t^{2}-1\right)\left(t^{2}-2\right)\left(t^{2}-3\right)\left(t^{2}-9\right) \cdots\left(t^{2}-\left(2^{k-1}+1\right)\right), \\
& p_{k}^{C}(t)=\alpha\left(t^{2}+1\right)\left(t^{2}-1\right)\left(t^{2}-2\right)\left(t^{2}-3\right)\left(t^{2}-9\right) \cdots\left(t^{2}-\left(2^{k-1}+1\right)\right), \\
& \tilde{p}_{k}^{C}(t)=\alpha\left(t^{2}-1\right)\left(t^{2}+1\right) t^{2}\left(t^{2}-7\right)\left(t^{2}-15\right) \cdots\left(t^{2}-\left(2^{k-1}-1\right)\right), \\
& \tilde{q}_{k}^{C}(t)=\alpha\left(t^{2}-1\right)\left(t^{2}+1\right) t^{2}\left(t^{2}-7\right)\left(t^{2}-15\right) \cdots\left(t^{2}-\left(2^{k-1}-1\right)\right) .
\end{aligned}
$$

\section{More details concerning $\Psi_{k}^{E}$}

This section is still concerned with determining the structure of the stabilizers. Specifically, we consolidate information about the element $\Psi_{k}^{E}$ that will be useful for the proof of $n$-transitivity presented in the next section. We are especially interested in how this element acts on large subsets of $\mathcal{C}_{2}$, which will appear later. The first result we need is the following formula for $2 \times 2$ matrices being raised to integer powers. It is an easy calculation by induction, and so we omit the proof.

Proposition 5. Let $M \in M_{2}(\mathbb{C})$ be an arbitrary matrix with two distinct eigenvalues. Let $\mu=\operatorname{Tr}(M)$ and $\nu=-\operatorname{det}(M)$. Then, for any $k \in \mathbb{Z}_{+}$, $M^{k}=\mu_{k} M+\nu \mu_{k-1} I$, where

$$
\mu_{k}=\frac{1}{\sqrt{\mu^{2}+4 \nu}}\left(\left(\frac{\mu+\sqrt{\mu^{2}+4 \nu}}{2}\right)^{k}-\left(\frac{\mu-\sqrt{\mu^{2}+4 \nu}}{2}\right)^{k}\right)
$$

for any $k \in \mathbb{Z}_{+}$.

We can now use this result to determine how $\Psi_{\gamma t^{2 n}}$ will act on points with $a_{2}=0$ :

Lemma 8. Applying $\Psi_{\gamma t^{2 n}}$ for any $n \in \mathbb{Z}_{+}$to the point $\left(a_{1}, 0, a_{3}, a_{4}, a_{5}\right)$, we arrive at the point $\left(a_{1}+\gamma a_{5}\left(\frac{a_{5}}{2}\right)^{n-1}, 0, a_{3}, a_{4}, a_{5}\right)$.

Proof. Let $A=\left(a_{1}, 0, a_{3}, a_{4}, a_{5}\right)$. We note that by definition, $a_{2}, a_{5}$ are fixed by the action of $\Psi$. Now consider $a_{1}$. Again by definition of $\Psi$, we know that

$$
a_{1}^{\prime}=\Psi_{\gamma t^{2 n}}\left(a_{1}\right)=a_{1}+\operatorname{Tr}\left(\gamma Y^{2 n}\right)=a_{1}+\gamma \operatorname{Tr}\left(Y^{2 n}\right) .
$$


If $a_{5} \neq 0$, we can now use the formula in Proposition 5 to get that $Y^{2 n}=\frac{a_{5}^{n}}{2^{n}} I$. Plugging into (7), we get

$$
a_{1}^{\prime}=a_{1}+\gamma \operatorname{Tr}\left(\frac{a_{5}^{n}}{2^{n}} I_{2}\right)=a_{1}+\frac{\gamma a_{5}^{n}}{2^{n-1}} .
$$

Next we consider the action of $\Psi_{\gamma t^{2 n}}$ on $a_{3}$ :

$$
\begin{aligned}
\Psi_{\gamma t^{2 n}}\left(a_{3}\right) & =a_{3}+2 \operatorname{Tr}(A q(Y))+\operatorname{Tr}\left(q^{2}(Y)\right)-\frac{1}{2} \operatorname{Tr}^{2}(q(Y)) \\
& =a_{3}+2 \operatorname{Tr}\left(A \cdot \frac{\gamma a_{5}^{n}}{2^{n}} I_{2}\right)+\operatorname{Tr}\left(\gamma^{2} \frac{a_{5}^{2 n}}{2^{2 n}} I_{2}\right)-\frac{1}{2} \operatorname{Tr}^{2}\left(\gamma \frac{a_{5}^{n}}{2^{n}} I_{2}\right) \\
& =a_{3}+\gamma^{2} \frac{a_{5}^{2 n}}{2^{2 n-1}}-\frac{1}{2}\left(\gamma \frac{a_{5}^{n}}{2^{n-1}}\right)^{2} \\
& =a_{3}+\gamma^{2} \frac{a_{5}^{2 n}}{2^{2 n-1}}-\frac{1}{2} \gamma^{2} \frac{a_{5}^{2 n}}{2^{2 n-2}} \\
& =a_{3},
\end{aligned}
$$

as claimed. We lastly consider the action on $a_{4}$ :

$$
\Psi_{\gamma t^{2 n}}\left(a_{4}\right)=a_{4}+\operatorname{Tr}\left(Y \cdot \gamma \frac{a_{5}^{n}}{2^{n}} I_{2}\right)=a_{4}+\gamma \frac{a_{5}^{n}}{2^{n}} \operatorname{Tr}(Y)=a_{4} .
$$

Thus, we see that $\Psi_{\gamma t^{2 n}}(A)=\left(a_{1}+\gamma \frac{a_{5}^{n}}{2^{n-1}}, 0, a_{3}, a_{4}, a_{5}\right)$, as desired.

If $a_{5}=0$, then we know that $Y$ is nilpotent, and hence $Y^{2 n} \equiv 0$ for all $n \in \mathbb{Z}_{+}$. It follows that $\Psi_{\gamma t^{2 n}}(A)=A$, so that the formula holds in this case as well.

Lemma 9. Applying $\Psi_{\gamma t^{2 n+1}}$ for any $n \in \mathbb{Z}_{+}$to the point $\left(a_{1}, 0, a_{3}, a_{4}, a_{5}\right)$, we arrive at the point

$$
\left(a_{1}, 0, a_{3}+\gamma^{2} \frac{a_{5}^{2 n+3}}{2^{2(n+1)}}, a_{4}+\gamma \frac{a_{5}^{n+2}}{2^{n+1}}, a_{5}\right) .
$$

Proof. The proof is analogous to that of Lemma 8.

Corollary 1. Let $A=\left(a_{1}, 0, a_{3}, a_{4}, a_{5}\right)$. Then $A^{\prime}=\Psi_{k}^{E}(A)$ (respectively, $A^{\prime}=\Psi_{k}^{O}(A)$ ) satisfies $a_{1}^{\prime} \equiv a_{1}$ (respectively, $a_{3}^{\prime} \equiv a_{3}$ ) if and only if $a_{5} \in R_{2}^{k}:=\left\{0,2,4, \ldots, 2^{k}\right\}$.

Proof. The proof is a straightforward application of Vieta's formula, using Lemma 8 (respectively, Lemma 9) and the fact that $\Psi_{f} \circ \Psi_{g}=\Psi_{f+g}$. 


\section{6. $\quad n$-Transitivity}

We now have everything we need in order to prove our main result: infinite-transitivity. We start with two supporting lemmas, analogous to Lemmas 1 and 2, and then proceed to the final theorem.

Notation. For any $k \in \mathbb{Z}_{+}$, we define the following notation:

$$
\begin{gathered}
C_{k}:=\left\{(0,0,0, \pm 1,0), \ldots,\left(0,0,0,-1,2^{k}\right)\right\} \backslash\{(0,0,0,-1,8)\} \subseteq \mathcal{C}_{2} ; \\
S_{k}:=\operatorname{Stab}\left[C_{k}\right] \subseteq G .
\end{gathered}
$$

Lemma 10. Let $A=\left(a_{1}, a_{2}, a_{3}, a_{4}, a_{5}\right) \in \mathcal{C}_{2} \backslash C_{k}$ for some $k \in \mathbb{Z}_{+}$. Then there is an element $g \in S_{k}$ such that $A^{\prime}=g(A)$ satisfies $a_{1}^{\prime} a_{3}^{\prime} \neq 0$.

Proof. Case 1: $a_{1} \neq 0$. If $a_{3} \neq 0$ as well, then we are done, so we assume $a_{3}=0$. Hence, the point is $A=\left(a_{1}, a_{2}, 0, \pm 1, a_{5}\right)$. Applying $\Phi_{-\frac{2 a_{2}}{a_{1}^{2}}-\frac{a_{1}^{2}}{4} \beta}$ to $A$, we arrive at the point $\left(a_{1}, 0,0, \pm 1, \mp \frac{4 a_{2}}{a_{1}}+a_{5} \pm \frac{1}{2} a_{1}^{3} \beta\right)$. Since $a_{1} \neq 0$ we can choose the parameter $\beta$ such that $a_{5} \notin R_{2}^{k}$. Then using Lemma 9 and Corollary 1 , we know that after applying $\Psi_{k}^{O}$, we will have that $a_{1}^{\prime}=a_{1} \neq 0$ and $a_{3}^{\prime}$ is non-constant polynomial in $\gamma$. Thus we can choose some $\gamma$ such that $a_{3}^{\prime} \neq 0$, as desired.

Case 2: $a_{3} \neq 0$. Similarly to Case 1, we assume that $a_{1}=0$, since otherwise we are done. Then, we apply the element $\Phi_{\alpha t^{2}}$ to arrive at the point

$$
A^{\prime}=\left(0, a_{2}+\alpha a_{3}, a_{3}, a_{4}, a_{5}\right) .
$$

We then see that

$$
\begin{aligned}
\left(a_{5}^{\prime}-\frac{1}{2}(\right. & \left.\left(2-3 a_{2}^{\prime 2}-2 \sqrt{1-2 a_{2}^{\prime 2}+2 a_{2}^{\prime 4}}\right)\right) \\
& \cdot\left(a_{5}^{\prime}-\frac{1}{2}\left(2-3 a_{2}^{\prime 2}+2 \sqrt{1-2 a_{2}^{\prime 2}+2 a_{2}^{\prime 4}}\right)\right) \\
=- & a_{2}^{2}+\frac{a_{2}^{4}}{4}-2 a_{5}+3 a_{2}^{2} a_{5}+a_{5}^{2}+\left(-a_{2} a_{3}+a_{2}^{3} a_{3}+6 a_{2} a_{3} a_{5}\right) \alpha \\
+ & \left(-a_{3}^{2}+\frac{3 a_{2}^{2} a_{3}^{2}}{2}+3 a_{3}^{2} a_{5}\right) \alpha^{2}+a_{2} a_{3}^{3} \alpha^{3}+\frac{a_{3}^{4} \alpha^{4}}{4} .
\end{aligned}
$$

Since $a_{3} \neq 0$, we conclude that the coefficient of $\alpha^{4}$ is non-zero, and hence this is a non-constant polynomial of $\alpha$. We then choose $\alpha \in \mathbb{C}$ such that

$$
a_{5}^{\prime}-\frac{1}{2}\left(2-3 a_{2}^{\prime 2} \pm 2 \sqrt{1-2 a_{2}^{\prime 2}+2 a_{2}^{\prime 4}}\right) \neq 0 .
$$


Then, we can apply the element $\Phi_{t^{4}-t^{2}}$ to $A^{\prime}$ to reach a point with

$$
\tilde{a}_{1}=\frac{1}{8}\left(a_{2}^{\prime 4}+4\left(-2+a_{5}^{\prime}\right) a_{5}^{\prime}+4 a_{2}^{\prime 2}\left(-1+3 a_{5}^{\prime}\right)\right) .
$$

This is zero if and only if $a_{5}^{\prime}=\frac{1}{2}\left(2-3 a_{2}^{\prime 2} \pm 2 \sqrt{1-2 a_{2}^{\prime 2}+2 a_{2}^{\prime 4}}\right)$, but by equation (8), we know this is not the case. Hence we are at a point with $\tilde{a}_{1} \neq 0$, so that we are back in Case 1 .

Case 3: $a_{1}=a_{3}=0$. Since $a_{3}=0$, we know that $a_{4}= \pm 1$ and at least one of $a_{2}, a_{5} \neq 0$, as otherwise the point would be nilpotent, and hence in $C_{k}$.

Case 3.1: $a_{5} \notin R_{2}^{k}$. Recall from Corollary 1 that $R_{2}^{k}=\left\{0,2,4, \ldots, 2^{k}\right\}$. If $a_{5} \notin R_{2}^{k}$, then this same corollary tells us that we can apply $\Psi_{k}^{E}$ for some value of $\alpha$ to get that $a_{1}^{\prime} \neq 0$. Then we are back in Case 1 .

Case 3.2: $a_{5} \in R_{2}^{k}$. This case requires the use of conjugation. For ease of notation, let $T=\left\{\left(0, a_{2}, 0, \pm 1, a_{5}\right)\right\}$ be the set of points that satisfy $a_{1}=a_{3}=0$.

Case 3.2.1: $a_{2} \neq 0$. Let $T_{0}=\left\{\left(0, a_{2}, 0, \pm 1, a_{5}\right)\right\}$ be the set of points we are considering here, so that $a_{2} \neq 0$ in $T_{0}$. We want to show that, using conjugation, we can move any element of $T_{0}$ to a point outside of $T$ (i.e. a point $A^{\prime}$ with $a_{1}^{\prime}$ or $\left.a_{3}^{\prime} \neq 0\right)$.

To do this, let $A=\left(0, a_{2}, 0,-1, a_{5}\right) \in T$ be an arbitrary point, and assume, for the sake of a contradiction, that none of the conjugations defined in section 4 move $A$ out of $T$. Then we have that for any choice of $\alpha$ in the polynomials $\tilde{q}_{k}^{C}$ and $\tilde{p}_{k}^{C}$, we must have that

$$
\left(\Phi_{t} \circ \Psi_{\tilde{q}_{k}^{C}} \circ \Phi_{t}\right)(A)=\left(0, a_{2}^{\prime}, 0, \pm 1, a_{5}^{\prime}\right) \in T
$$

and

$$
\left(\Psi_{-t} \circ \Phi_{\tilde{p}_{k}^{C}} \circ \Psi_{t}\right)(A)=\left(0, \hat{a}_{2}, 0, \pm 1, \hat{a}_{5}\right) \in T .
$$

Moving $\Phi_{t}$ and $\Psi_{t}$ to the other side of the equations, we see that these reduce to

$$
\begin{gathered}
\Psi_{\tilde{q}_{k}^{C}}\left(0, a_{2}, 0,-1, a_{5}-2\right)=\left(0, a_{2}^{\prime}, 0, \pm 1, a_{5}^{\prime} \pm 2\right) \\
\Phi_{\tilde{p}_{k}^{C}}\left(a_{2}, a_{2}, a_{5}-2,-1+a_{5}, a_{5}\right)=\left(\hat{a}_{2}, \hat{a}_{2}, \hat{a}_{5} \pm 2, \pm 1+\hat{a}_{5}, \hat{a}_{5}\right) .
\end{gathered}
$$

We will use these equations to prove the following claim:

Claim 1. Let $C_{Y}(t)$ denote the characteristic polynomial of $Y$. Then we have that $C_{Y}(t) \mid \tilde{q}_{k}^{C}(t)$. 
For now, we will assume this claim is true. This implies, since $\tilde{p}_{k}^{C}(t)=$ $\tilde{q}_{k}^{C}(t)$, that the eigenvalues of $X$ and $Y$ are roots of $\tilde{p}_{k}^{C}(t)$.

We now consider the element $\Psi_{k}^{E}$. We know from Lemma 8 and Corollary 1 that $\Psi_{k}^{E}$ fixes the point $A$. Thus, using the definition of the action by $\Psi_{q}$, we see that $\operatorname{Tr}\left(q_{k}^{E}(Y)\right)=\Psi_{k}^{E}\left(a_{1}\right)=0$ and $2 \operatorname{Tr}(A$. $\left.q_{k}^{E}(Y)\right)+\operatorname{Tr}\left(q_{k}^{E}(Y)^{2}\right)=\Psi_{k}^{E}\left(a_{3}\right)=0$. This is true for all $\alpha$, and hence, since $2 \operatorname{Tr}\left(A \cdot q_{k}^{E}(Y)\right)$ is linear in $\alpha$ while $\operatorname{Tr}\left(q_{k}^{E}(Y)^{2}\right)$ is quadratic in $\alpha$, we must have that their coefficients are 0 separately. In particular, we get that $\operatorname{Tr}\left(q_{k}^{E}(Y)\right)=0$ and $\operatorname{Tr}\left(q_{k}^{E}(Y)^{2}\right)=0$, so that $q_{k}^{E}(Y)$ is nilpotent, and hence the eigenvalues of $q_{k}^{E}(Y)$ are both 0 . If we denote the eigenvalues of $Y$ by $\lambda_{1}, \lambda_{2}$, then we obtain the fact that $q_{k}^{E}\left(\lambda_{1}\right)=0=q_{k}^{E}\left(\lambda_{2}\right)$ for all $\alpha \in \mathbb{C}$, so that $\lambda_{1}$ and $\lambda_{2}$ are roots of $q_{k}^{E} / \alpha$.

Then, combining this with the preceding statement, we see that the eigenvalues $\lambda_{1}$ and $\lambda_{2}$ are roots of both

$$
\begin{gathered}
q_{k}^{E}(t) / \alpha=t^{2(k+1)}+S(1, k-1) t^{2 k}+\cdots+S(k-1, k-1) t^{4}+S(k, k-1) t^{2} ; \\
\tilde{p}_{k}^{C}(t) / \alpha=\left(t^{2}-1\right)\left(t^{2}+1\right) t^{2}\left(t^{2}-7\right)\left(t^{2}-15\right) \cdots\left(t^{2}-\left(2^{k-1}-1\right)\right) .
\end{gathered}
$$

The only roots these equations share are given by 0 and \pm 1 , and since $\tilde{p}_{k}^{C}$ has no double roots, we know that $\lambda_{1} \neq \lambda_{2}$. Additionally, since $\lambda_{1}+\lambda_{2}=$ $\operatorname{Tr}(Y)=a_{2} \neq 0$ by the case assumption, we also know that $\lambda_{1} \neq-\lambda_{2}$. Thus we can conclude that if both $\left(\Phi_{-t} \circ \Psi_{\tilde{q}_{k}^{C}} \circ \Phi_{t}\right)(A) \in T$ and $\left(\Psi_{-t} \circ\right.$ $\left.\Phi_{\tilde{p}_{k}^{C}} \circ \Psi_{t}\right)(A) \in T$, then $A$ satisfies $a_{2}= \pm 1$.

Running the same argument with $\Phi_{t} \circ \Psi_{q_{k}^{C}} \circ \Phi_{-t}$ and $\Psi_{t} \circ \Phi_{p_{k}^{C}} \circ \Psi_{-t}$, we get that if both $\left(\Phi_{t} \circ \Psi_{q_{k}^{C}} \circ \Phi_{-t}\right)(A) \in T$ and $\left(\Psi_{t} \circ \Phi_{p_{k}^{C}} \circ \Psi_{-t}\right)(A) \in T$, then $A$ must satisfy $a_{2} \in\{1 \pm \sqrt{2},-1 \pm \sqrt{2}\}$.

Since, by assumption we have that all of these conjugations land $A$ back in $T$, we thus conclude that $a_{2} \in\{ \pm 1\}$ and $a_{2} \in\{1 \pm \sqrt{2},-1 \pm \sqrt{2}\}$, so that $a_{2} \in\{ \pm 1\} \cap\{1 \pm \sqrt{2},-1 \pm \sqrt{2}\}$ which is a contradiction. Thus, we must have that at least one of the conjugations moves the point $A$ out of the set $T$, thus landing us back in a previous case where $a_{1} \neq 0$ or $a_{3} \neq 0$. An analogous argument gives the same result if we start with a point $A$ where $a_{4}=1$.

Case 3.2.2: $a_{2}=0$. Since we also have that $a_{1}=a_{3}=0$, in order for our point $A$ to not be $C_{k}$, we see that we are reduced to considering the set of points

$$
\begin{gathered}
\{(0,0,0,1,2),(0,0,0,1,4),(0,0,0, \pm 1,8) \\
\left.(0,0,0,1,16), \ldots,\left(0,0,0,1,2^{k}\right)\right\}
\end{gathered}
$$


We will now show that using the element $\Psi_{-t} \circ \Phi_{\tilde{p}_{k}^{C}} \circ \Psi_{t}$, we can move all of these points out of this set. To do this, we first note that applying any number of times repeatedly gives a conjugation with $\Phi_{n \tilde{p}_{k}^{C}}$ in the middle. Now let $A$ be a point in (11), and assume that $\left(\Psi_{-t} \circ \Phi_{n \tilde{p}_{k}^{C}} \circ \Psi_{t}\right)(A)$ is in (11) for all $n \in \mathbb{Z}_{+}$. Then, since there are only finitely many points in (11), this means that there must be some $n, m \in \mathbb{Z}_{+}$with $m>n$ such that $\left(\Psi_{-t} \circ \Phi_{n \tilde{p}_{k}^{C}} \circ \Psi_{t}\right)(A)=\left(\Psi_{-t} \circ \Phi_{m \tilde{p}_{k}^{C}} \circ \Psi_{t}\right)(A)=\left(\left(\Psi_{-t} \circ \Phi_{(m-n) \tilde{p}_{k}^{C}} \circ \Psi_{t}\right) \circ\right.$ $\left.\left(\Psi_{-t} \circ \Phi_{n \tilde{p}_{k}^{C}} \circ \Psi_{t}\right)\right)(A)$. In particular, we see that $\Psi_{-t} \circ \Phi_{(m-n) \tilde{p}_{k}^{C}} \circ \Psi_{t}$ fixes the point $\left(\Psi_{-t} \circ \Phi_{n \tilde{p}_{k}^{C}} \circ \Psi_{t}\right)(A)$ in (11). However, this is a contradiction to Lemma 6 , which guarantees that no points in (11) are fixed by conjugation of the form $\Psi_{-t} \circ \Phi_{n \tilde{p}_{k}^{C}} \circ \Psi_{t}$ for $n \in \mathbb{Z}_{+}$. Thus we must have that successive usage of the element $\Psi_{-t} \circ \Phi_{\tilde{p}_{k}^{C}} \circ \Psi_{t}$ will move any point in (11) out of this set and into a previous case.

To finish the proof, we need to prove Claim 1.

Proof of Claim 1 We start by showing that $a_{2}=a_{2}^{\prime}=\hat{a}_{2}$ and $a_{5}=a_{5}^{\prime}=$ $\hat{a}_{5}$. To do this, we first consider equation (9). Since $\Psi$ fixes $a_{2}$ and $a_{5}$, this equation tells us immediately that $a_{2}=a_{2}^{\prime}$. Thus we only need to consider $a_{5}$. There are two cases:

$$
\begin{gathered}
\Psi_{\tilde{q}_{k}^{C}}\left(0, a_{2}, 0,-1, a_{5}-2\right)=\left(0, a_{2}^{\prime}, 0,1, a_{5}^{\prime}+2\right) ; \\
\Psi_{\tilde{q}_{k}^{C}}\left(0, a_{2}, 0,-1, a_{5}-2\right)=\left(0, a_{2}^{\prime}, 0,-1, a_{5}^{\prime}-2\right) .
\end{gathered}
$$

If the first of these is true, then we must have, by examining the 4th term, that $\operatorname{Tr}\left(B \cdot \tilde{q}_{k}^{C}(Y)\right)=2$ for that specific choice of $\alpha$, and if the second is true we must have that, for those specific $\alpha \in \mathbb{C}$, that $\operatorname{Tr}\left(B \cdot \tilde{q}_{k}^{C}(Y)\right)=0$. We easily see from the definition of $\tilde{q}_{k}^{C}$ that if $\alpha=0$, then $\operatorname{Tr}\left(B \cdot \tilde{q}_{k}^{C}(Y)\right)=0$. This implies either that, as a function of $\alpha$, we have that $\operatorname{Tr}\left(B \cdot \tilde{q}_{k}^{C}(Y)\right) \equiv 0$ or $\operatorname{Tr}\left(B \cdot \tilde{q}_{k}^{C}(Y)\right)$ is non-constant. If the second of these options is true, then we can choose some $\alpha \in \mathbb{C}$ such that $\operatorname{Tr}\left(B \cdot \tilde{q}_{k}^{C}(Y)\right) \neq 0,2$. However, for such an $\alpha$, we then have that $a_{4}^{\prime} \neq \pm 1$, which contradicts the fact that we must have $a_{3}^{\prime} a_{5}^{\prime}+a_{4}^{\prime 2}=1$. Thus we must have that $\operatorname{Tr}\left(B \cdot \tilde{q}_{k}^{C}(Y)\right) \equiv 0$, so that $a_{5}-2=a_{5}^{\prime}-2$ for all $\alpha \in \mathbb{C}$. Thus we conclude that $a_{5}=a_{5}^{\prime}$, as claimed. An analogous argument using equation (10) gives that $a_{2}=\hat{a}_{2}$ and $a_{5}=\hat{a}_{5}$.

Thus, using the second equation above that we have found to be the case, we must have that

$$
\Psi_{\tilde{q}_{k}^{C}}\left(0, a_{2}, 0,-1, a_{5}-2\right)=\left(0, a_{2}, 0,-1, a_{5}-2\right)
$$


so that $\Psi_{\tilde{q}_{k}^{C}}$ fixes the point $\left(0, a_{2}, 0,-1, a_{5}-2\right)$. We can then write that $\tilde{q}_{k}^{C}(t)=C_{Y}(t) f(t)+r(t)$, where $r(t)$ has degree $\leqslant 1$, since $C_{Y}(t)$ has degree 2. However, since Cayley-Hamilton guarantess that $C_{Y}(Y)=0$, we conclude that $\tilde{q}_{k}^{C}(Y)=r(Y)$, so that $\Psi_{\tilde{q}_{k}^{C}}=\Psi_{r}$, and hence $\Psi_{r}$ fixes the point $\left(0, a_{2}, 0,-1, a_{5}-2\right)$. However, it is easy to see that the only polynomial of degree less than or equal to 1 that fixes this point is the zero polynomial, and hence $r(t) \equiv 0$. This shows that $C_{Y}(t) \mid \tilde{q}_{k}^{C}(t)$, as claimed.

We now move onto the second lemma that will be necessary in proving infinite-transitivity.

Lemma 11. Let $A \in \mathcal{C}_{2} \backslash C_{k}$ for some $k \in \mathbb{Z}_{+}$satisfy $a_{1} a_{3} \neq 0$. Then there is a $g \in S_{k} \subseteq G$ such that $A^{\prime}=g(A)$ satisfies both $a_{1}^{\prime} a_{3}^{\prime} \neq 0$ and $a_{1}^{\prime 4}-4 a_{3}^{\prime 2} \neq 0$.

Proof. We may assume that $a_{1}^{2}-4 a_{3}^{2}=0$, since otherwise we are done.

Case 1: $a_{1}^{2}=-2 a_{3}$. Applying the element $\Phi_{\gamma t^{2}+\beta t^{4}}$ with $\beta=-\frac{2 a_{2}}{a_{1}^{4}}$ we have that $a_{2}^{\prime}=0$ and

$$
a_{5}^{\prime}=a_{5}+a_{1} \gamma\left(2 a_{4}-\frac{a_{1}^{3} \gamma}{2}\right)=a_{5}+2 a_{1} a_{4} \gamma-\frac{a_{1}^{4} \gamma^{2}}{2}
$$

while $a_{1}, a_{3}$ stay fixed. We note that since $a_{1} \neq 0$, the coefficient of $\gamma^{2}$ is non-zero, and hence $a_{5}^{\prime}$ is a non-constant polynomial in $\gamma$, so that we can choose some $\gamma \in \mathbb{C}$ such that $a_{5}^{\prime} \notin\left\{0,2,4, \ldots, 2^{k}\right\}$. Let $\tilde{A}=\Psi_{k}^{E}\left(A^{\prime}\right)$. Using Lemma 8 and Corollary 1 , we know that $\tilde{a}_{1}$ is a non-constant polynomial in $\alpha$ while $\tilde{a}_{3}=a_{3}=\frac{a_{1}^{2}}{2}$ is non-zero and constant. Thus $\tilde{a}_{1}^{2} \pm 2 \tilde{a}_{3}$ will be a non-constant polynomials in $\alpha$, since a constant function cannot cancel higher order non-constant terms. Thus we can choose some $\alpha \in \mathbb{C}$ such that $\tilde{a}_{1} \tilde{a}_{3} \neq 0$ and $\tilde{a}_{1}^{2} \pm 2 \tilde{a}_{3} \neq 0$, as desired.

Case 2: $a_{1}^{2}=2 a_{3}$. We start at the point $A=\left(a_{1}, a_{2}, \frac{a_{1}^{2}}{2}, a_{4}, a_{5}\right)$. Consider the polynomial

$$
\begin{aligned}
q(t) & =t^{2}\left(t^{2}-1\right)\left(t^{2}-2\right)\left(t^{2}-4\right) \cdots\left(t^{2}-2^{k-1}\right) \\
& =t^{2(k+1)}+S(1, k-1) t^{2 k}+\cdots S(k-1, k-1) t^{4}+S(k, k-1) t^{2}
\end{aligned}
$$

and recall that, by construction, $\Psi_{\alpha q(t)}$ will stabilize $C_{k}$ for any $\alpha \in \mathbb{C}$.

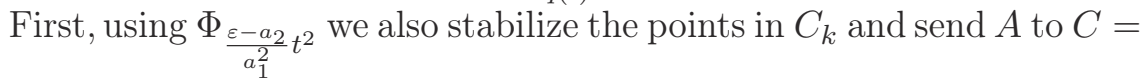
$\left(a_{1}, \varepsilon, \frac{a_{1}^{2}}{2}, a_{4}^{\prime}, a_{5}^{\prime}\right)$, where $a_{4}^{\prime}=a_{4}+\frac{\varepsilon-a_{2}}{2} a_{1}$ and $a_{5}^{\prime}=a_{5}+\frac{\left(\varepsilon-a_{2}\right)^{2}}{2}+\frac{2 a_{4}\left(\varepsilon-a_{2}\right)}{a_{1}}$. 
Now, for $\Psi_{\alpha q(t)}(C)=\left(a_{1, \alpha}, a_{2, \alpha}, a_{3, \alpha}, a_{4, \alpha}, a_{5, \alpha}\right)$, we calculate the following (keep in mind that this family of automorphisms fixes points in $C_{k}$ ):

$$
\begin{gathered}
a_{1, \alpha}=\alpha \cdot \operatorname{Tr}(q(Y))+a_{1}, \\
a_{3, \alpha}=\alpha^{2} \cdot\left(\operatorname{Tr}\left(q^{2}(Y)\right)-\frac{1}{2} \operatorname{Tr}^{2}(q(Y))\right)+2 \alpha \cdot \operatorname{Tr}(A q(Y))+\frac{a_{1}^{2}}{2}
\end{gathered}
$$

Our goal is to show that there exists a nonzero $\alpha$ such that $a_{3, \alpha}=-\frac{1}{2} a_{1, \alpha}^{2}$ with $a_{1, \alpha} \neq 0$, since we will then be back in Case 1. Let $f(\alpha)=a_{3, \alpha}+\frac{1}{2} a_{1, \alpha}^{2}$. We now need to find the roots of $f(\alpha)$.

$$
f(\alpha)=\alpha^{2} \cdot \operatorname{Tr}\left(q^{2}(Y)\right)+\alpha\left(2 \operatorname{Tr}(A q(Y))+a_{1} \operatorname{Tr}(q(Y))\right)+a_{1}^{2} .
$$

Let $\alpha_{1}$ and $\alpha_{2}$ be roots of $f(\alpha)$. Then both of them are not zero since $a_{1} \neq 0$. Since $\operatorname{Tr}(Y)=\varepsilon$ and $\operatorname{Tr}\left(Y^{2}\right)=\operatorname{Tr}\left(B^{2}\right)+\frac{1}{2} \operatorname{Tr}^{2}(Y)=a_{5}^{\prime}+\frac{1}{2} \varepsilon^{2}$ we find the eigenvalues of $Y$ to be given by $\mu_{1}=\frac{\varepsilon}{2}+\frac{\sqrt{2 a_{5}^{\prime}}}{2}$ and $\mu_{2}=\frac{\varepsilon}{2}-\frac{\sqrt{2 a_{5}^{\prime}}}{2}$.

We also have that

$$
\operatorname{Tr}(q(Y))=q\left(\mu_{1}\right)+q\left(\mu_{2}\right) \text { and } \operatorname{Tr}\left(q^{2}(Y)\right)=q^{2}\left(\mu_{1}\right)+q^{2}\left(\mu_{2}\right),
$$

so we can calculate that

$$
\begin{aligned}
& \operatorname{Tr}(q(Y))=q\left(\mu_{1}\right)+q\left(\mu_{2}\right) \\
& =\mu_{1}{ }^{2(k+1)}+\sum_{i=1}^{k} \alpha_{i} \mu_{1}{ }^{2 i}+\mu_{2}{ }^{2(k+1)}+\sum_{i=1}^{k} \alpha_{i} \mu_{2}{ }^{2 i} \\
& =\mu_{1}{ }^{2(k+1)}+\mu_{2}{ }^{2(k+1)}+\sum_{i=1}^{k} \alpha_{i}\left(\mu_{1}{ }^{2 i}+\mu_{2}{ }^{2 i}\right) \\
& =\left(\frac{\varepsilon}{2}+\frac{\sqrt{2 a_{5}^{\prime}}}{2}\right)^{2(k+1)}+\left(\frac{\varepsilon}{2}-\frac{\sqrt{2 a_{5}^{\prime}}}{2}\right)^{2(k+1)} \\
& \quad+\sum_{i=1}^{k} \alpha_{i}\left(\left(\frac{\varepsilon}{2}+\frac{\sqrt{2 a_{5}^{\prime}}}{2}\right)^{2 i}+\left(\frac{\varepsilon}{2}-\frac{\sqrt{2 a_{5}^{\prime}}}{2}\right)^{2 i}\right) \\
& \left.=\frac{1}{2^{k+1}}\left(\varepsilon^{2}+2 \varepsilon \sqrt{2 a_{5}^{\prime}}+2 a_{5}^{\prime}\right)^{(k+1)}+\left(\varepsilon^{2}-2 \varepsilon \sqrt{2 a_{5}^{\prime}}+2 a_{5}^{\prime}\right)^{(k+1)}\right) \\
& \quad+\sum_{i=1}^{k} \frac{\alpha_{i}}{2^{2 i}}\left(\left(\varepsilon^{2}+2 \varepsilon \sqrt{2 a_{5}^{\prime}}+2 a_{5}^{\prime}\right)^{i}+\left(\varepsilon^{2}-2 \varepsilon \sqrt{2 a_{5}^{\prime}}+2 a_{5}^{\prime}\right)^{i}\right) .
\end{aligned}
$$

From this we know $\operatorname{Tr}(q(Y))$ is a polynomial in $\varepsilon$ of degree $2(k+1)$; call it $g(\varepsilon)$. 
Similarly, we get that

$$
\begin{array}{r}
\operatorname{Tr}\left(q^{2}(Y)\right)=\frac{1}{4^{k+1}}\left(\left(\varepsilon^{2}+2 \varepsilon \sqrt{2 a_{5}^{\prime}}+2 a_{5}^{\prime}\right)^{2(k+1)}+\left(\varepsilon^{2}-2 \varepsilon \sqrt{2 a_{5}^{\prime}}+2 a_{5}^{\prime}\right)^{2(k+1)}\right) \\
+\sum_{i=1}^{2 k+1} \frac{\beta_{i}}{4^{2 i}}\left(\left(\varepsilon^{2}+2 \varepsilon \sqrt{2 a_{5}^{\prime}}+2 a_{5}^{\prime}\right)^{i}+\left(\varepsilon^{2}-2 \varepsilon \sqrt{2 a_{5}^{\prime}}+2 a_{5}^{\prime}\right)^{i}\right) .
\end{array}
$$

This implies that $\operatorname{Tr}\left(q^{2}(Y)\right)$ is a polynomial in $\varepsilon$ of degree $4(k+1)$, say $h(\varepsilon)$.

Let us show that for either $\alpha=\alpha_{1}$ or $\alpha=\alpha_{2}$, we must have that $a_{1, \alpha} \neq 0$. If $a_{1, \alpha}$ is zero in both cases, it implies that

$$
0=-\left(2 \operatorname{Tr}(A q(Y))+a_{1} \operatorname{Tr}(q(Y))\right) \operatorname{Tr}(q(Y))+2 a_{1} \operatorname{Tr}\left(q^{2}(Y)\right) .
$$

This shows that the discriminant of $f(\alpha)$ (say $D$ ) is zero, i.e. $D=0$. Since we can choose $\varepsilon$ such that $g(\varepsilon) h(\varepsilon) \neq 0$, we then get that

$$
\begin{gathered}
\left(2 \operatorname{Tr}(A q(Y))+a_{1} \operatorname{Tr}(q(Y))\right)=\frac{2 a_{1} \operatorname{Tr}\left(q^{2}(Y)\right)}{\operatorname{Tr}(q(Y))}, \\
0=D=\frac{4 a_{1}^{2} \operatorname{Tr}\left(q^{2}(Y)\right)\left(\operatorname{Tr}\left(q^{2}(Y)\right)-\operatorname{Tr}^{2}(q(Y))\right)}{\operatorname{Tr}^{2}(q(Y))}
\end{gathered}
$$

We have

$$
\begin{aligned}
& \left.\operatorname{Tr}\left(q^{2}(Y)\right)-\operatorname{Tr}^{2}(q(Y))\right)=-2 q\left(\mu_{1}\right) q\left(\mu_{2}\right) \\
& =\left(\mu_{1}^{2(k+1)}+\sum_{i=1}^{k} \alpha_{i} \mu_{1}^{2 i}\right)\left(\mu_{2}^{2(k+1)}+\sum_{i=1}^{k} \alpha_{i} \mu_{2}^{2 i}\right) \\
& =\left(\mu_{1} \mu_{2}\right)^{2(k+1)}+\sum_{i=1}^{k} \alpha_{i} \mu_{1}^{2 i} \mu_{2}^{2(k+1)}+\sum_{i=1}^{k} \alpha_{i} \mu_{2}^{2 i} \mu_{1}^{2(k+1)} \\
& +\sum_{i=1}^{k} \alpha_{i} \mu_{1}^{2 i} \sum_{i=1}^{k} \alpha_{i} \mu_{2}^{2 i} .
\end{aligned}
$$

From this it is not difficult to see that this is a polynomial in $\varepsilon$, which we will denote $x(\varepsilon)$. Taking $\varepsilon$ such that $g(\varepsilon) h(\varepsilon) x(\varepsilon) \neq 0$, we reach a contradiction. Thus, using the above actions, we can arrive at the point $\left(b_{1}, \varepsilon,-\frac{b_{1}^{2}}{2}, b_{4}, b_{5}\right)$ with $b_{1} \neq 0$; we then use the proof of Case 1 to achieve the desired result.

Finally, we can prove infinite transitivity:

Theorem 1. The action of $G$ on $\mathcal{C}_{2}$ is a n-transitive group action for all $n \in \mathbb{Z}_{+}$, and hence infinitely transitive. 
Proof. Let $A \in \mathcal{C}_{2} \backslash C_{k}$ be an arbitrary point. To prove the theorem, it is sufficient to show that there is a $g \in S_{k}$ such that $g(A)=\left(0,0,0,-1,2^{k+1}\right)$. Using Lemmas 10 and 11, we may assume that $a_{1} a_{3} \neq 0$ and $a_{1}^{2} \pm 2 a_{3} \neq 0$. Then, since $\Phi_{p} \in S_{k}$ when $p(t)=\alpha t^{2}+\beta t^{3}$, we can let

$$
\begin{aligned}
\alpha=\frac{2}{a_{3}\left(a_{1}^{2}+2 a_{3}\right)\left(a_{1}^{2}-2 a_{3}\right)^{4}}\left(3 a_{1}^{8} a_{2} a_{3}-4 a_{1}^{6} a_{2} a_{3}^{2}-16 a_{1}^{4} a_{2} a_{3}^{3}+16 a_{1}^{2} a_{2} a_{3}^{4}\right. \\
+16 a_{2} a_{3}^{5}-a_{1}^{9} a_{4}-4 a_{1}^{7} a_{3} a_{4}+16 a_{1}^{5} a_{3}^{2} a_{4}+16 a_{1}^{3} a_{3}^{3} a_{4}-48 a_{1} a_{3}^{4} a_{4} \\
\left.\quad+\left(a_{1}^{3}+6 a_{1} a_{3}\right) \sqrt{\left(a_{1}^{2}-2 a_{3}\right)^{4}\left(a_{1}^{2}+2 a_{3}\right)^{2}\left(1+2^{k+1} a_{3}\right)}\right)
\end{aligned}
$$

and

$$
\begin{gathered}
\beta=\frac{4}{a_{3}\left(a_{1}^{2}-2 a_{3}\right)^{4}}\left(2 a_{1}^{5} a_{2} a_{3}-8 a_{1}^{3} a_{2} a_{3}^{2}+8 a_{1} a_{2} a_{3}^{3}-a_{1}^{6} a_{4}+2 a_{1}^{4} a_{3} a_{4}\right. \\
\left.+4 a_{1}^{2} a_{3}^{2} a_{4}-8 a_{3}^{3} a_{4}+\sqrt{\left(a_{1}^{2}-2 a_{3}\right)^{4}\left(a_{1}^{2}+2 a_{3}\right)^{2}\left(1+2^{k+1} a_{3}\right)}\right)
\end{gathered}
$$

to move $A$ to the point

$$
A^{\prime}=\Phi_{p}(A)=\left(a_{1}, 0, a_{3}, a_{4}^{\prime}, 2^{k+1}\right)=\left(a_{1}, 0, \frac{a_{4}^{\prime 2}-1}{2^{k+1}}, a_{4}^{\prime}, 2^{k+1}\right) .
$$

Then, letting $q(t)=\sum_{i=2}^{m} \beta_{i} t^{i}$, we will show that $\Psi_{q} \in S_{k}$.

We first write $q(t)=\sum_{i=2}^{\left[\frac{m}{2}\right]} \beta_{2 i} t^{2 i}+\sum_{i=1}^{\left[\frac{m}{2}\right]-1} \beta_{2 i+1} t^{2 i+1}$. Then by Lemma 8 and Lemma 9 we get that

$$
\left\{\begin{array}{l}
\Psi_{q}(0,0,0,1,0)=(0,0,0,1,0), \\
\Psi_{q}(0,0,0,-1,0)=(0,0,0,1,0), \\
\Psi_{q}\left(0,0,0,-1,2^{j}\right)=\left(\sum_{i=2}^{\left[\frac{m}{2}\right]} 2^{i(j-1)+1} \beta_{2 i}, 0, \sum_{i=1}^{\left[\frac{m}{2}\right]-1} \beta_{2 i+1}^{2} 2^{2 i(j-1)+3 j-2},\right. \\
\left.\quad \sum_{i=1}^{\left[\frac{m}{2}\right]-1} \beta_{2 i+1} 2^{i(j-1)+2 j-1}, 2^{j}\right), \quad j=\overline{1, k}, j \neq 3, \\
\Psi_{q}\left(a_{1}, 0, \frac{a_{4}^{\prime 2}-1}{2^{k+1}}, a_{4}, 2^{k+1}\right)=\left(a_{1}+\sum_{i=2}^{\left[\frac{m}{2}\right]} 2^{k i+1} \beta_{2 i}, 0,\right. \\
\left.\quad \frac{a_{4}^{\prime 2}-1}{2^{k+1}}+\sum_{i=1}^{\left[\frac{m}{2}\right]-1} \beta_{2 i+1}^{2} 2^{2 k(i+1)+k+1}, a_{4}^{\prime}+\sum_{i=1}^{\left[\frac{m}{2}\right]-1} \beta_{2 i+1} 2^{k(i+2)}, 2^{k+1}\right) .
\end{array}\right.
$$


From these we obtain the following systems:

$$
\begin{gathered}
\left\{\begin{array}{l}
\sum_{i=2}^{\left[\frac{m}{2}\right]} 2^{i(j-1)} \beta_{2 i}=0 \quad j=\overline{1, k}, j \neq 3 \\
\sum_{i=2}^{\left[\frac{m}{2}\right]} 2^{k i+1} \beta_{2 i}=-a_{1}
\end{array}\right. \\
\left\{\begin{array}{l}
\sum_{i=1}^{\left[\frac{m}{2}\right]-1} \beta_{2 i+1} 2^{i(j-1)+2 j-1}=-1 \\
\sum_{i=1}^{\left[\frac{m}{2}\right]-1} \beta_{2 i+1} 2^{k(i+2)}=-1-a_{4}^{\prime}
\end{array} \quad j=\overline{1, k}, j \neq 3\right.
\end{gathered}
$$

We need to show the systems (12) and (13) have solutions. For the systems (12) and (13) we have the following matrix:

$$
\left(\begin{array}{cccccc}
1 & 1 & 1 & 1 & \ldots & 1 \\
1 & 2 & 2^{2} & 2^{3} & \ldots & 2^{k-1} \\
1 & 2^{3} & 2^{6} & 2^{9} & \ldots & 2^{3(k-1)} \\
1 & 2^{4} & 2^{8} & 2^{12} & \ldots & 2^{4(k-1)} \\
\vdots & \vdots & \vdots & \vdots & \ddots & \vdots \\
1 & 2^{k} & 2^{2 k} & 2^{3 k} & \ldots & 2^{k(k-1)}
\end{array}\right)
$$

The determinant of this matrix is Vandermonde's determinant and is not zero. Thus we can choose coefficients so that $\Psi_{q} \in S_{k}$, and applying this element to $A^{\prime}$, we get that $\Psi_{q}\left(A^{\prime}\right)=\left(0,0,0,-1,2^{k+1}\right)$. Therefore, all elements in $\mathcal{C}_{2} \backslash C_{k}$ are in the same orbit as $\left(0,0,0,-1,2^{k+1}\right)$ under the action of $S_{k}$, and hence $G$ acts $n$-transitively on $\mathcal{C}_{2}$, as desired.

\section{Conclusion}

While Berest-Eshmatov-Eshmatov's conjecture has been recently proved, our approach for the case $n=2$ brings more clarity of the action on $\mathcal{C}_{2}$, which could be useful for future studies.

\section{References}

[1] Yu. Berest, A. Eshmatov, F. Eshmatov, Multitransivity of Calogero-Moser spaces. Transform. Groups 21 (2016), 35-50.

[2] V. Drensky, E. Formanek, Polynomial Identity Rings, Adv. Courses Math. CRM Barcelona, Birkhäuser, Basel, 2004.

[3] K. Kuyumzhiyan, Infinite transitivity for Calogero-Moser spaces, Proc. Amer. Math. Soc., posted on 2020, DOI:10.1090/proc/15030; arXiv:1807.05723.

[4] G. Wilson, Collisions of Calogero-Moser particles and an adelic Grassmannian (with an Appendix by I. G. Macdonald), Invent. Math. 133 (1998), 1-41.

[5] W. Van der Kulk, On polynomial rings in two variables, Nieuw Arch. Wisk., 1 (1953), 33-41. 
CONTACT INFORMATION

Jacob Kesten

Department of Mathematics, Rice University, Houston, TX, 77005, USA

E-Mail(s): jgk3@rice.edu

Samuel Mathers Department of Mathematics, Princeton

University, Princeton, NJ, 08544, USA

E-Mail(s): smathers@princeton.edu

Zafar Normatov V. I. Romanovskiy Institute of Mathematics, Uzbekistan Academy of Sciences, Tashkent, 100170, Uzbekistan

E-Mail(s): z.normatov@mathinst.uz

Received by the editors: 26.06.2020

and in final form 05.12.2020. 